\title{
LANDCOVER CHANGE MODELING THROUGH VISUALIZATION AND CLASSIFICATION ENHANCEMENT OF MULTI-TEMPORAL IMAGERY
}

\author{
PARTSINEVELOS P. ${ }^{1}$ \\ NIKOLAKAKI N. ${ }^{1}$ \\ PSILLAKIS P. ${ }^{1}$ \\ MILIARESIS G. ${ }^{2}$ \\ XANTHAKIS M. ${ }^{3}$
}

\author{
${ }^{1}$ School of Mineral Resources Engineering \\ Technical University of Crete, Chania, Greece \\ ${ }^{2}$ Open University of Cyprus \\ Environmental Conservation \& Management \\ ${ }^{3}$ Management Body of Mt. Ainos National Park \\ Cephalonia, Greece
}

Received: $24 / 09 / 2014$

Accepted: $18 / 02 / 2015$

*to whom all correspondence should be addressed:

Available online: $17 / 03 / 2015$ e-mail: ppartsinevelos@isc.tuc.gr

\section{ABSTRACT}

The natural environment endures natural and humaninduced changes. Remote sensing has been providing monitoring oriented solutions by a series of methodologies which contribute to prudent environmental management. Analysis of multi-temporal satellite images for the observation of the land changes often includes error prone classification and changedetection techniques. The present study takes advantage of the temporal continuity of multi-temporal classified images, in order to model change trends and reduce classification uncertainty, based on reasoning rules. By revealing misclassification prone areas, training site selection is targeted. Moreover, computational tools are developed in order to disclose the alterations in land use dynamics and offer spatial reference to the pressures

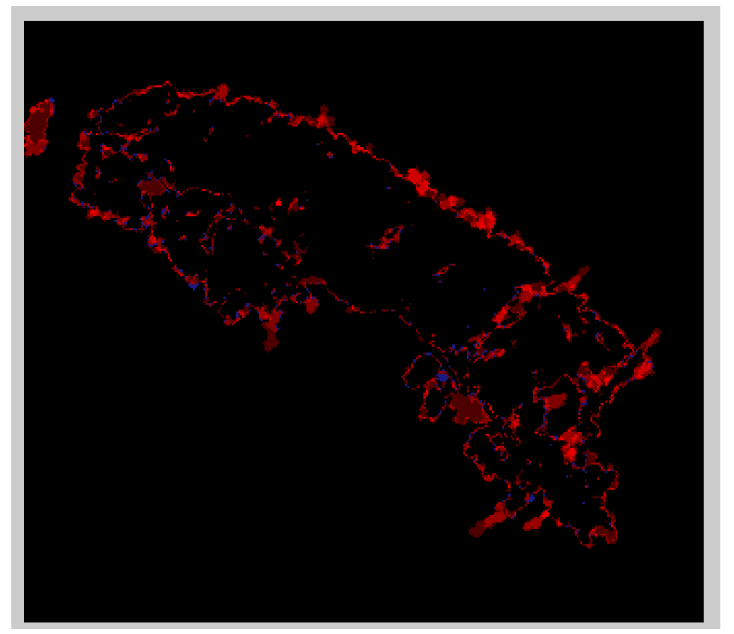

Multi-temporal classification error map that land use classes impose. The proposed procedures are tested upon Landsat time series imagery, depicting the National Park of Ainos in Kefallinia, Greece.

Keywords: classification, land use/land change, multi-temporal imagery, change detection

\section{Introduction}

The automatic or semi-automatic detection of changes over time through satellite imagery spans in a wide range of applications such as monitoring of natural phenomena (e.g. forest fires, floods, earthquakes) and the assessment of anthropogenic activities (e.g. vegetation, agricultural production, urban development). Remote sensing, has been providing monitoring oriented solutions regarding the natural Earth surface, by offering a series of tools and methodologies which contribute to the sustainable environmental management (Lillesand et al., 2008). The natural habitat constantly endures both inherent natural and human-induced influences. The continuous monitoring of these influences, through land-use alterations, offers remarkable advantages for the determination of actions regarding the management and protection of endangered areas (Styers et al., 2009; Otukei and Blascke, 2010; Coppin et al., 2004). 
Usually, the identification of land use changes initially involves the classification of multi-temporal satellite images (Petropoulos, 2012). Apart from classification, processing and analysis of multi-temporal satellite images for the observation of the land use/land changes includes most often change-detection practices. Multi-temporal imagery may be processed through visual interpretation of temporally plotted indexes of classified image areas (Chen et al., 2005). As seen in Bovolo and Bruzzone, (2007), the magnitude of spectral change based on temporally registered pixels provides an estimation of significant change. Typical image classification includes pixel based and object based techniques under several variations of classification algorithms. (Campbell, 2011). In Etteieb et al., 2012, forest mapping classification, under pixel and object based techniques, is discussed. In the present study a change based technique is proposed that encompasses time to provide possible misclassification areas in the form of clustered pixel regions. Thus, pixel based classification is performed, yet, object based inference is used to classify change areas. Other pixel or region based techniques include robust satellite techniques (Aliano et al., 2008) and cross correlation analysis.

Related studies under an object oriented image perspective, involve classification and change detection (Zhou et al., 2008; Lizarazo and Elsner, 2009). Studies include object identification with the help of already classified regions from a GIS (Walter, 2004), segmentation upon object attributes (Reinhold and Selsam, 2010), intensity correlation in bi-temporal images (Jensen and Tullis, 2008) and hierarchical mapping of geo-objects (Handcock and Csillag, 2004; Johansen et al., 2010; Chen et al., 2012).

Classification and change detection remain error prone procedures since they are influenced mainly by the distinctive characteristics of the study areas, the remote sensing systems limitations, the existing conditions during image acquisition and the image processing and image analysis methodologies and algorithms used. Standard techniques for ground reference training pixel selection include random, stratified, random equalized and stratified random techniques. (Hashemian et al., 2004). Sampling and validation is not always reliable (Foody 2002; Grenier et al., 2008). Generally, in forestry applications similar schemes are used (Martin et al., 1998; Suárez et al., 2005). Land change and forest applications can be based on existing maps that may carry their own errors (Fuller et al., 2003; Charou et al., 2012). In this study, error prone training areas are proposed to the classifier in order to prevent classification errors.

Accordingly, visualization and synthesis of change detection results are often described by simple percentages or become too complex to be comprehended from a non-expert, while important information is obscured. Moreover, commercially available images are not often affordable for small scale applications. Nevertheless, free imagery, because of the relatively low resolution (e.g. $30 \mathrm{~m}$ ), does not always provide optimal classification results.

Towards this end, the present study takes advantage of the temporal continuity of the classified images, in order to improve the results of the classification, based on reasoning rules upon the segmented objectsclasses. Moreover, since many well known Remote Sensing commercial software packages display solely quantitative alteration in the land use changes, tools are developed in order to disclose the alterations in land use changes and offer a spatial reference to the pressures that the classes impose between them. The developed algorithms are tested upon multi-temporal Landsat satellite imagery depicting the National Park of Ainos in Kefallinia, while the results are directly applicable to similar types of applications and satellite image time series. The results have shown that the use of the above suggested procedures can contribute towards the advancement of the classification procedure of satellite images and support the existing knowledge regarding the localization and assessment of land-use changes.

The main objective of this study is to find areas that are susceptible to misclassification, yet additional objectives are considered. Though the study, training site selection during the process of supervised classification is proposed in order to provide better differentiation between classification classes. Another underlying objective is to contribute to the understanding and analysis of anthropogenic and environmental factors that influence land use changes. Overall, the objectives of the study include:

- Improvement of the classification accuracy,

- Provision of a base model to extract trends of change, 
- Spatial referencing of significant changes in land use,

- Visualization of spatiotemporal pressure for single or in-between classes,

- Improvement of training area selection for classification

\section{Methodology-Approach}

The input datasets for the corresponding processes include the multi-temporal classified imagery for the application area, along with the classified area types. The proposed approach includes processes for the spatiotemporal pressure endured by a single class, or class pairs and the object-based error prone delineation of the classification results through spatiotemporal inference.

\subsection{Spatiotemporal visualization of intra and inter-class pressures}

Change detection often provides a series of quantitative and map results to demonstrate the changes occurred between classes through two different time instances. Assessment of change detection results is often complex for the non-expert decision maker, since it either lacks spatial reference, or is demonstrated at its whole, making the resulting map incomprehensible. Accordingly, by exploiting multitemporal images the above mentioned difficulties are multiplied. Yet, multi-temporal change detection can provide valuable information by portraying selective, combinational and distinct spatiotemporal information.

Towards this end, the process first focuses on portraying a single class and visualize its alterations through time. This simple procedure includes sequential subtraction of the subsequent time series classification images upon a selected class. Thus, changes of a single class through time are depicted in a new image where growth or reduction of the specific class is indicated. The algorithm simply adds the first segmented image to the chronologically future segmented image multiplied by a factor of two, and thus assigning a distinctive number to each relation category, namely unchanged, growth and reduction. According to the magnitude and/or direction of the change which is regarded as important, the visualization indicates areas of significant change. For computational efficiency the searching algorithm does not sequentially visit all the pixels, yet according to change information it may skip several positions when pixel alteration is minimal. Temporal increments may selectively include any number and any temporal step throughout the time series. Under this procedure the user can understand the evolution of a single class in terms of change, yet the rest of the classes are not revealed.

Accordingly, a second algorithm associates pairs of segmented classification classes to portray inter-class comparison. The aim is to show the effects that each class undergoes from a neighboring class. To differentiate the change values three relation categories are introduced, namely, unchanged, " $A$ " class infected " $B$ " class, and " $A$ " class infected by "B" class. Again, significant information in terms of size can be included in the visualization, along with selective temporal pairing. As stated before, change detection map complexity rises exponentially when classes, temporal instances, and change types rise. Thus, visualization of intra and inter change for a single or a pair of classes provides a solid and comprehensive visualization alternative.

\subsection{Classification accuracy enhancement}

According to Anderson et al. (1976), there is no ideal classification of land cover and it is unlikely that one could ever be developed. That is because patterns of land cover change according to the needs for natural resources. Each classification is based on user requirements and few users will be satisfied by a list that would cover most requirements. Moreover, in image classification there is no established, secure way to evaluate the classification accuracy by conventional validation point assessment. In addition, common change detection algorithms are performed through subtraction of classification maps and thus inherit errors from both classifications. In order to reduce classification errors, temporal continuity of the image time series is taken into consideration. The classified image is considered as a set of segmented objects and algorithmic inference provides possible classification errors. Thus, areas that exhibit a random behavior through time are detected, e.g. oscillate between classification classes. In other words, when 
areas appear and disappear through the time series in an erratic fashion, then these areas are suspect to misclassification.

To implement the proposed process, first an assignment of sub-class areas for all classified images takes place, through an equivalent in terms of size circular shape. Next, the algorithm identifies for each area how many times it appears in which images of the time series, by comparing the distances of the centers and number of pixels for each individual sub-area. The process compares a region of the first image in relation to all areas of the rest of the images. Then, an automatic reference counter, records how many times a change happens. To facilitate the procedure, a map table records the change and matching regions. Each row in the table refers to a region, and each column in an image of the time series. Binary values indicate whether a sub-region is evident (1) or not (0) in each time frame.

If a class change appears on one frame and continues to show as change, then this change is bound to be valid. If a changing area fluctuates between classes or disappears and reappears in an erratic manner then this change area is prone to misclassification. Generally, if a change is made more than once, then it is likely an error. Using the above mentioned table it is now simple to map which sub-regions are evident and when by simply adding each row. Additionally, the table facilitates a quick reference to extract trends of change. Based on simple algorithms one may focus on specified changes according to the application at hand. E.g. if the temporal increment is known and the time series is long enough, phenology of vegetation may be modeled and tagged as important or non-important change. Any periodic or systematic type of change can be modeled and queried upon the table.

An additional raster visual representation, counts how many times a pixel or a segmented group of pixels changes throughout the time series. The end result is an image showing the "variability" of pixels mapped in a particular color map. The image has the same size with the original ones, and includes values from 0 to $n-1$, where $n$ is the number of images in the time series. Thus, in a single image, the whole time series change behavior is mapped portraying in a glance possible classification errors.

Thus, an attempt to detect errors resulting from the process of classification by means of logic rules which record the occasional misclassified groups of pixels into classes is presented. The users may take the possible classification errors into account, by targeting specific regions when selecting training samples. In addition the user may fine tune the classification procedure even by introducing new classes.

\section{Study area, data and pre-processing}

To demonstrate the applicability of the proposed method, a case study of a forest area concerning land use change was undertaken. The case study area is the Hellenic National Park of Ainos in Cephalonia (figure 1). The National Park consists of an area of 2.862 ha, which expand at the mountain Ainos and at the adjacent mountain Roudi. Mt. Ainos with an alt. of $1,628 \mathrm{~m}$ is the tallest mountain in the lonian Islands, whereas Mt. Roudi reaches an alt. of $1,125 \mathrm{~m}$. The park extends in longitude between $20^{\circ} 37^{\prime}$ and $20^{\circ} 44^{\prime}$ and in latitude between $38^{\circ} 05^{\prime}$ and $38^{\circ} 11^{\prime} 06$.

Its main feature is its Cephalonian fir forest (Abiescephalonica), which is unique in the lonian Islands. Abiescephalonica is a Greek endemic species, i.e. it occurs only in the Greek region. In particular, it occurs in Cephalonia, Evvia island and in mainland Greece from Peloponnesus to Mt. Olympus and Mt. Athos, at an altitude of 600-1,600 m (Farjon, 2010). The need to protect the Fir forest on Mt. Ainos, where it was described as a new species (locus classicus), as well as the phytogeographical importance of its presence in Cephalonia, constituted the main reasons that resulted in the declaration of Mt. Ainos as a National Park in 1962. In previous centuries, cephalonian fir was an important tree species particularly after the 16th century. This fact led to extensive woodcutting in the forest of Mt. Ainos-Mt. Roudi, as well as the inability to effectively control and put out fires that occurred in the area, threatened the survival of the Fir forest. As a result, its distribution area has been restricted to $1 / 4$ of the initial one (Samios, 1908). Moreover, during the last 5 decades a visible decrease in Greek fir populations has been reported for Greece (Raftoyannis and Radoglou 2001; Tsopelas et al. 2001; Politi et al., 2009). 


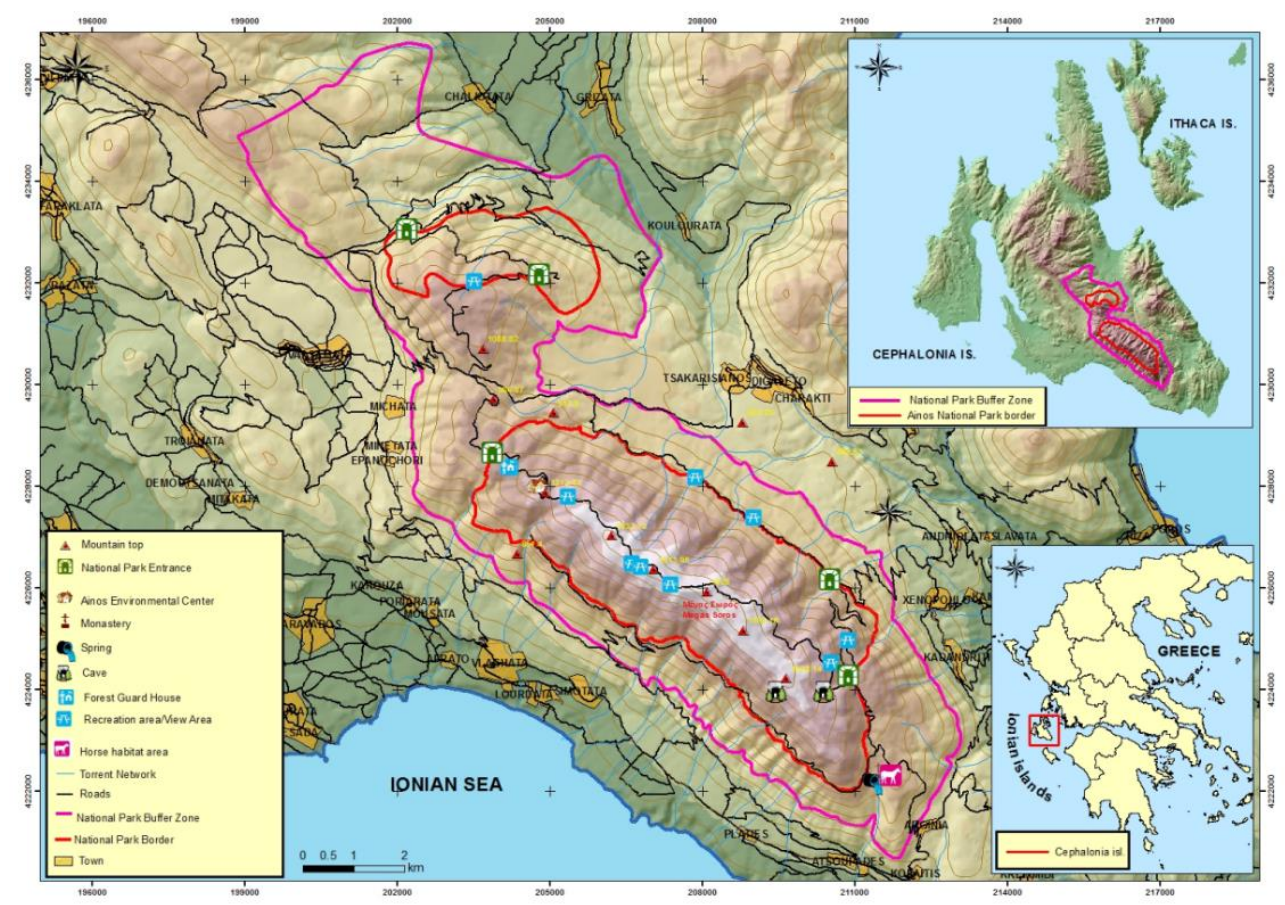

Figure 1. Study area of the Hellenic National Park of Mt. Ainos

In recognition of its significance at the European level, the National Park has been designated as a European Biogenetic Reserve, it belongs to the "Natura 2000" European Ecological Network of Protected Areas and is a Special Protected Area for the protection of the avifauna. It has also been declared as a Wildlife Reserve.

Due to free availability, the preferred satellite imagery originated from Landsat 5 and Landsat 7 missions. The final selection of satellite images was performed under various criteria, including: clarity, absence of clouds, reduced stripping, and coherent time periods and epochs in order to reduce differences in the appearance of vegetation, which would result in variations in the process of classification. Eight images were selected, from 2000 to 2011 preferably during the summer months.
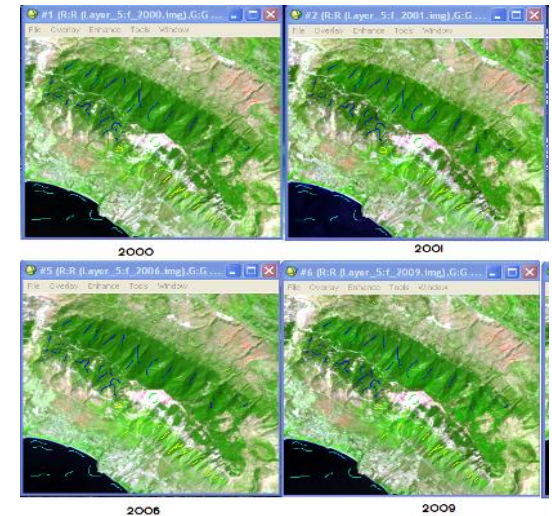

(a)

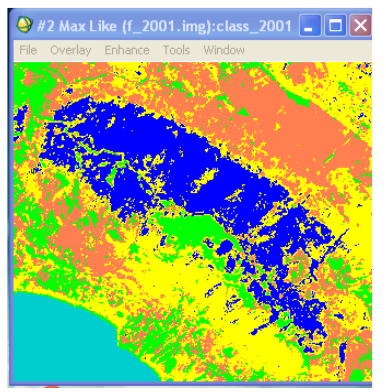

(b)

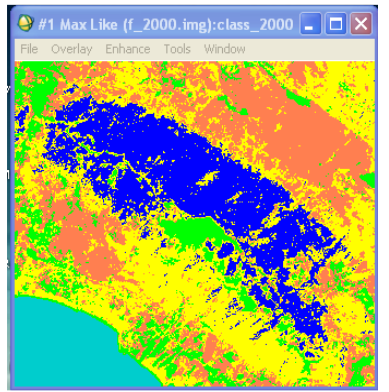

Figure 2. Sample of a) case study region selection for four time instances, b) classification results

The whole procedure was performed through ENVI and ERDAS IMAGINE software. The images were of "L1T" processing level so they have been under radiometric and geometric corrections. The image preprocessing included geo-referencing, conversion of brightness values to radiance units and selection of the area of interest. In addition, in order to compensate for radiometric variations due to image 
acquisition conditions, histogram matching was performed. Finally, the spectral bands were combined under layer stacking (figure 2a).

For the present study the Maximum Likelihood supervised classification method was used (fiure $2 \mathrm{~b}$ ) since it presented better preliminary results from other traditional classification algorithms. The selection criterion was visual consistency and durability of classes in relation with known land cover data (photos, etc).Minimum distance algorithm was also used as proposed for land cover change applications, but it was verified that the maximum likelihood algorithm gave more accurate results (Inzana et al. 2003; Jobin et al., 2010). The designated land cover categories included Abiescephalonica, Water, Limestone, Vegetation, Soil, based on the following criteria; a) literature data, b) Corine classification, c) information from foresters of the Management Body of Mt. Ainos National Park in Cephalonia. In order to perform the supervised classification, selection of class types and training-validation areas took place. Along the same lines, training samples were selected with the assistance of the Corine 2000 land use cover, Google Earth, several points from in situ information with GPS coordinates.

Next, change detection tools were used to identify changes between the eight classified images. As mentioned previously, this technique is the most common change detection technique, but inherits the possible errors from both image classifications. At this stage, regular change detection methodology would proceed to the justification and interpretation of the results based on quantified percentages of changes and the change maps. One has to be cautious since interpretation in the corresponding scale may prove misleading, since errors in classification are inherited in change detection in the same areas where change is anticipated, e.g. the borders between classes.

\section{Results}

The following experimental results use developed algorithms in Matlab upon the classified time series processed for the National Park of Ainos. The results are differentiated according to temporal inclusion (use of two or more time frames), for one or more classes (intra and inter class), and towards classification error detection.

According to intra-class spatiotemporal change shown in figure 3 , the significant changes are depicted as growth (blue areas) or reduction (red areas).

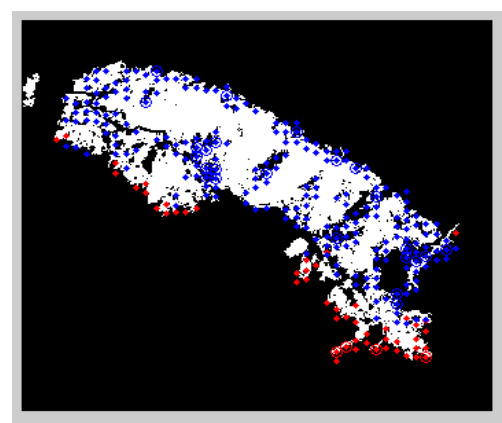

Figure 3. Intra-class change detection through time for the class Abiescephalonica

In figure 4, the combination of two classes portrays the pressure under any temporal increment. E.g.as seen in figure 4a, the class vegetation and soil between 2006 and 2009 show small changes on the borders of the classes. Yet, the same comparison between 2009 and 2010 yields a large scale difference in the central south region of figure $4 \mathrm{~b}$. After detecting this abrupt change, it was confirmed that a fire took place eleven days before the image acquisition in Simotata region. Finally, in figure 4c the comparison resumes between 2010 and 2011 where no apparent change in vegetation verifies no reclamation or classification error.

Investigation of the change instances through the change matrix presented in section 2 shows that region 1 of image 1 is present in all the time series except time 6 . Accordingly, region number 3 is shown only in 
time 3 and then disappears. Again, every matrix row corresponds to a sub-region and every column to the instance of the time series. According to the application at hand, these changes are possible trends or errors. In our case they are considered as errors and validation of the classification results is performed according to the new findings. With the classic validation point classification error estimation no difference was determined, thus, visual investigation for the above mentioned areas proved that they were actually classification errors.

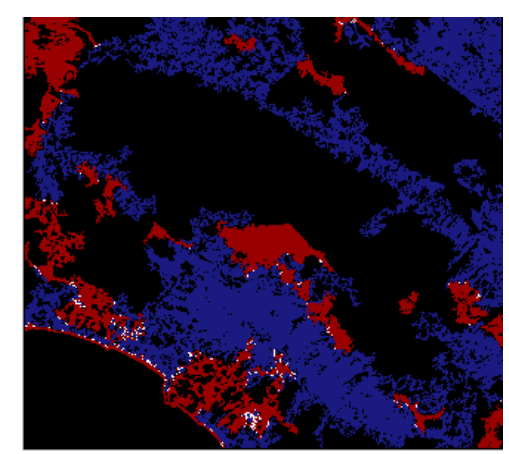

(a)

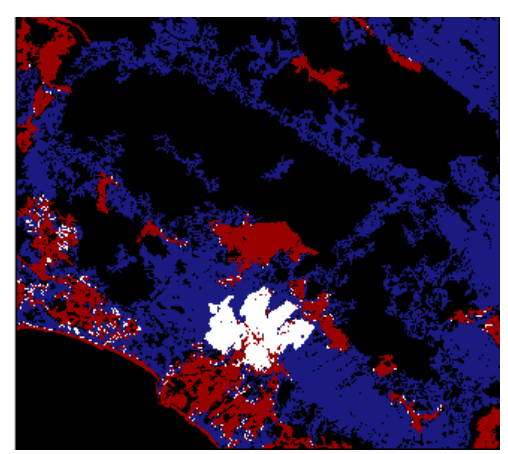

(b)

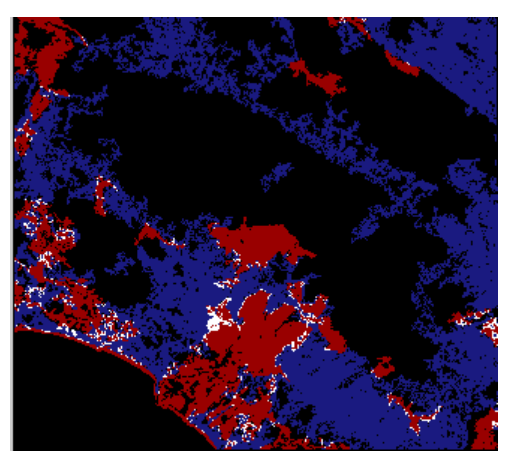

(c)

Figure 4. Inter-class spatiotemporal pressures between vegetation and soil classes

Accumulated changes from the whole time series can be overlaid, as shown in figure 6 . An overall change image is automatically drawn given the input data of the multi-temporal classified images. As seen in figure 5 the black color corresponds to no change, the blue to single change and the variations of red gradually portray the remaining five changes. Through this single image the error prone areas can be easily detected.

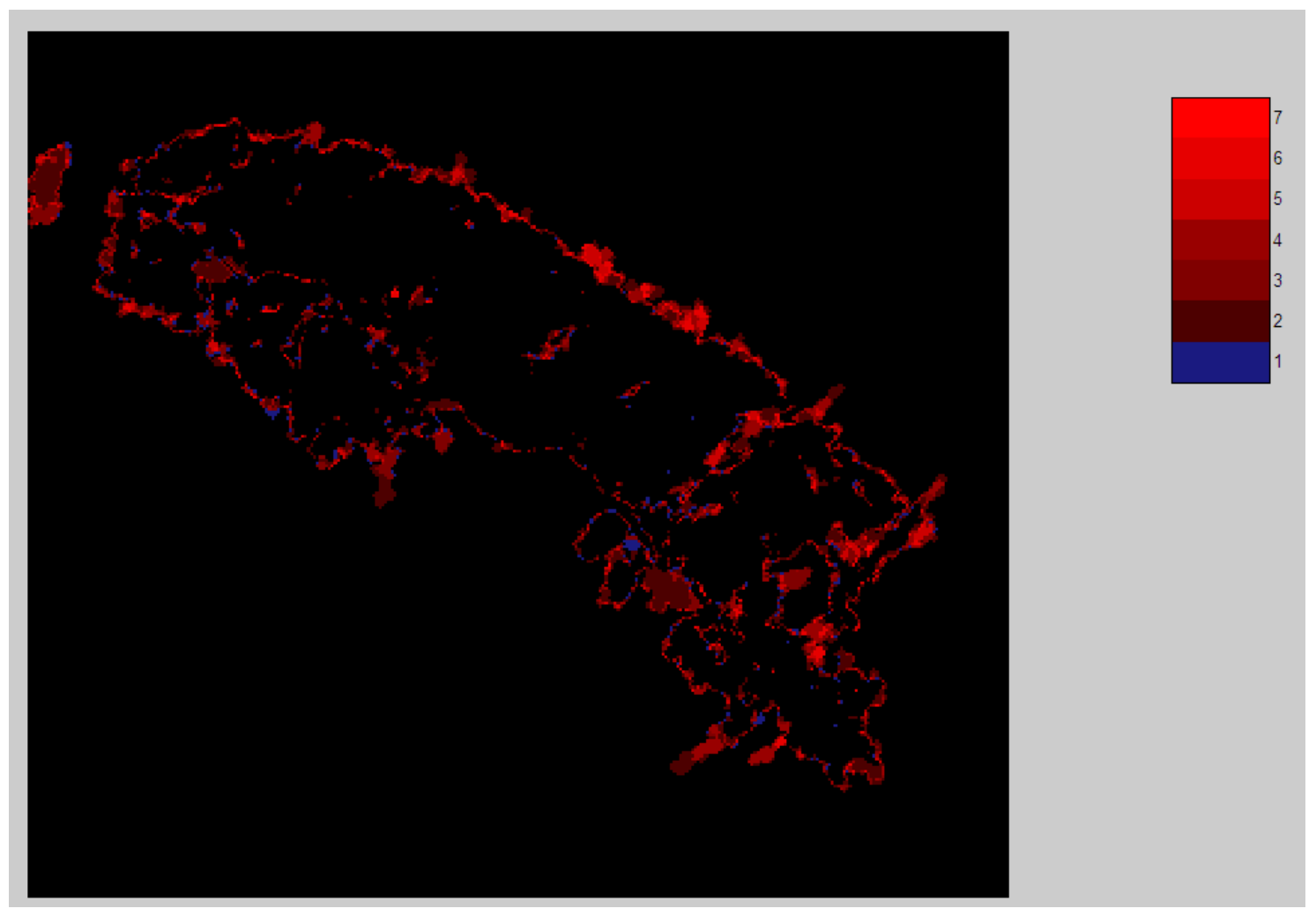

Figure 5. Accumulated raster change image 
It is evident that most changes occur upon the borders of the national park. It is an application related procedure to investigate whether this change is a classification error or an actual change. Towards this end, further GIS based processes may take place. E.g. if one is sure that border change is not significant then the border of the park area can be masked out.

\section{Discussion of Results}

Despite the large number of change detection techniques that have been developed to date, they inevitably inherit errors that occur during classification. Overall, the results have shown that the use of the suggested procedures contributed to the reduction of the classification uncertainty. There is no secure way to evaluate the classification accuracy by conventional validation point assessment. In order to improve accuracy in classification, we take advantage of temporal continuity of the images and detect areas that exhibit a random behavior through time by oscillating between classification classes.

Apart from the size of change which is detected through the intra and interclass relations, importance may refer to the type of change. The proposed change matrix is a simple yet valuable base model to query various types of systematic change trends. E.g. "find areas that change every August", or "find areas with semi-annual periodicity", or "find areas that move randomly", etc.

Moreover, by revealing areas that are susceptible to misclassification, specific target site selection for training during the process of supervised classification is facilitated. Along with the minor changes and pressures indicated in the test area due to harvesting and other human interventions, the developed algorithms successfully captured a fire incident that has been historically confirmed.

Overall, according to the fulfilled objectives of the study, it has been shown that there was an improvement on classification accuracy and corresponding training area selection. Spatiotemporal reference of changes provided a change sensitivity map upon which misclassification can be detected. Concurrently, class behavior in time is visualized in terms of single class dynamics or two class interaction. Finally, a base model for trend identification is proposed for further analysis.

\section{Conclusions}

The natural environment, because of the factors affecting life cycle, whether they are natural (e.g. climate change) or man-made (e.g. structures), normally suffers changes with various levels of intensity. Monitoring change through land use changes can cast some light to the real causes of change, in order to take appropriate measures for prevention, protection and response. The developed tools, by differentiating the class combinations in time under various spatiotemporal scales, facilitate decision making and understanding of change.

This study proposes a series of tools to assist satellite image change detection in two ways. First, it aids change comprehension through the spatiotemporal visualization of intra and inter-class relations. Second, it uses inference to assume possible classification errors. Thus, pixel and object based techniques were utilized to portray change information and enhance classification results. More specifically, a change based method is proposed that incorporates time and inference to provide clustered pixel groups prone to error. By selecting as validation and training data the error prone areas a robust and reliable training and validation is performed.

The findings and developed tools are directly applicable to similar types of applications and satellite image time series. The results have shown that the use of the suggested procedures can support the existing knowledge regarding the localization of land-use changes.

In the future a quantitative assessment of validation and training selection would be automated and a new validation scheme and training set selection algorithms is to be proposed. Finally, a trend vocabulary can be structured to describe the various behaviors that change reveals, when class changes occur in space and time. 


\section{References}

Aliano C., Corrado R., Filizzola C., Pergola N. and Tramutoli V. (2008), Robust Satellite Techniques (RST) for the thermal monitoring of earthquake prone areas: the case of Umbria-Marche October, 1997 seismic events, Annals of Geophysics, 51(2/3), 451-459.

Bovolo F. and Bruzzone L. (2007), A Theoretical Framework for Unsupervised Change Detection Based on Change Vector Analysis in Polar Domain, IEEE Transactions on Geoscience and Remote Sensing, 45(1), 218-236.

Campbell J.B. (2011). Introduction to Remote Sensing, 5th edition. New York. The Guilford Press. ISBN 9781609181765.

Charou E., Stefouli M., Petridis S., Poirazidis K. and Martinis A. (2012), Multitemporal Remote Sensing Data Analysis of Land Cover Changes of the Ionian Islands, Greece", European Association of Remote Sensing Laboratories Special Interest Group (SIG) Temporal Analysis of Satellite Images, MYKONOS, Greece.

Chen G., Hay G.J., Carvalho L.M.T. and Wulder M.A. (2012), Object-based change detection, International Journal of Remote Sensing, 33, 14, 4434-4457.

Chen X., Vierling L., Deering D. and Conley A. (2005), Monitoring boreal forest leaf area index across a Siberian burn chronosequence: a MODIS validation study, International Journal of Remote Sensing, 26(24), 5433-5451.

Coppin P., Jonckheere I., Nackaerts K. and Muys B. (2004), Digital change detection methods in ecosystem monitoring: a review, International Journal of Remote Sensing, 38, 2017-2031.

Etteieb S., Louhaichi M., Kalaitzidis C. and Gitas I.Z. (2012), Mediterranean forest mapping using hyper-spectral satellite imagery, Arab J Geosci., doi: 10.1007/s12517-012-0748-6.

Farjon A., (2010), A handbook of the world's conifers, Vol. 1, Koninklijke Brill NV, Leiden, The Netherlands.

Foody G.M. (2002), Status of land cover classification accuracy assessment, Remote Sensing of Environment, 80, 185-201.

Fuller R.M., Smith G.M. and Devereux B.J. (2003), The characterization and measurement of land cover change through remote sensing: problems in operational applications?, International Journal of Applied Earth Observation and Geoinformation, 4, 243-253.

Grenier M., Labrecque S., Benoit M. and Allard M. (2008), Accuracyassessmentmethodfor wetland object-based classification. In: Proceedings GEOBIA, 2008, Pixels,Objects, Intelligence: GEOgraphic Object Based Image Analysis for the 21stCentury. pp. 285-289.

Handcock R.N. and Csillag F. (2004), Spatio-temporal analysis using a multiscale hierarchical ecoregionalization, Photogrammetric Engineering and Remote Sensing, 70, 101-110.

Hashemian M.S., Abkar A.A. and Fatemi S.B. (2004), Study of Sampling Methods for Accuracy Assessment of Classified remotely Sensed Data, International congress for photogrammetry and remote sensing; ISPRS XXth congress, 1682-1750.

Inzana J., Kusky T., Higgs G. and Tucker R. (2003), Supervised classifications of Landsat TM band ratio images and Landsat TM band ratio image with radar for geological interpretations of central Madagascar, Journal of African Earth Sciences, 37, 59-72.

Jobin B., Latendresse C., Grenier M., Maisonneuve C. and Sebbane A. (2010), Recent Landscape Change at the Ecoregion Scale in Southern Québec (Canada) 1993-2001, Environmental Monitoring Assessement, 164(1-4), 631-647, DOI 10.1007/s10661-009-0918-5.

Johansen, Kasper, Arroyo, Lara A., Phinn, Stuart and Witte, Christian (2010). Comparison of geo-object based and pixel-based change detection of riparian environments using high spatial resolution multi-spectral imagery. In: Russell G. Congalton, Special Issue on Geographic Object-Based Image Analysis (GEOBIA). GEOBIA 2008: Pixels, Objects, Intelligence. GEOgraphic Object Based Image Analysis for the 21st Century, Calgary, Alberta, Canada, (123-136). 5-8 August 2008.

Lillesand T., Kiefer R. and Chipman J. (2008), Remote sensing and image interpretation, John Wiley \& Sons, New York, $768 \mathrm{p}$.

Lizarazo I. and Elsner P. (2009), Fuzzy segmentation for object-based image classification, International Journal of Remote Sensing, 30(6), 1643-1649.

Martin M.E., Newman S.D., Aber J.D. and Congalton R.G. (1998), Determining Forest Species Composition Using High Spectral Resolution Remote Sensing Data, Remote Sensing of Environment, 65(3), 249-254, ISSN 0034-4257. 
Otukei J.R. and Blaschke T. (2010), Land cover change assessment using decision trees, support vector machines and maximum likelihood classification algorithms, International Journal of Applied Earth Observation and Geoinformation, 12, Supplement 1, S27-S31.

Petropoulos G.P., Partsinevelos P. and Mitraka Z. (2012), Change detection of surfacemining activity and reclamation based on a machine learning approach of multi-temporal Landsat TM imagery, Geocarto International, DOI:10.1080/10106049.2012.706648.

Politi P.I., Arianoutsou M. and Stamou G.P. (2009), Patterns of Abiescephalonica seedling recruitment in Mount Aenos National Park, Cephalonia, Greece, For. Ecol. Manag., 258, 1129-1136.

Raftoyannis Y. and Radoglou K. (2001), Crown condition of a fir forest in Karpenisi, Central Greece.In: International Conference on Forest Research "A challenge for an integrated European approach", NAGREF, Forest Research Institute Thessaloniki, Greece, 317-320.

Reinhold M. and Selsam P. (2010), Automated Change Detection for Thematic Data Using Object-Based Analysis of Remote Sensing Imagery, In: Geographic Object-Based Image Analysis, 29 June - 2 July, 2010, Ghent, Belgium, Editor(s): E.A. Addink and F.M.B. Van Coillie

Samios K.M., 1908. The forests of Kefallinia. National Publications Office, Athens, pp.120-131 (in Greek).

Styers D.M., Chappelka A.H., Marzen L.J. and Somers G.L. (2009), Developing a land-cover classification to select indicators of forest ecosystem health in a rapidly urbanizing landscape, Landscape and Urban Planning, 94(3-4), doi:10.1016/j.landurbplan.2009.09.006.

Suárez J.C., Smith S., Bull G., Malthus T.J., Donoghue D. and Knox D. (2005), The Use of Remote Sensing Techniques in Operational Forestry, Quarterly Journal of Forestry, 99(1), 31-42.

Tsopelas P., Angelopoulos A., Economou A., Voulala M. and Xanthopoulou E. (2001), Monitoring crown defoliation and tree mortality in the fir forest of Mount Parnis, Greece. In: International Conference on Forest Research "A challenge for an integrated European approach". NAGREF, Forest Research Institute Thessaloniki, Greece, 253 258.

Walter V. (2004), Object-based classification of remote sensing data for change detection, ISPRS Journal of Photogrammetry \& Remote Sensing, 58, 225- 238.

Zhou W., Troy A. and Grove J.M. (2008), Object-based land cover classification and change analysis in the Baltimore metropolitan area using multi-temporal high resolution remote sensing data, Sensors, 8, 1613-1636. 